\title{
Condutividade elétrica em sementes de abóbora, híbrido Bárbara
}

\author{
Roberval Daiton Vieira ${ }^{1}$; Alek Sandro Dutra ${ }^{2}$ \\ ${ }^{1}$ UNESP-FCAV-Dep ${ }^{\text {to }}$. Produção Vegetal, 14884-900 Jaboticabal-SP; ${ }^{2}$ Bolsista do CNPq/DCR/FUNCAP, UFC-Dep ${ }^{\text {to }}$. Fitotecnia, \\ Fortaleza-CE; E-mail: rdvieira@fcav.unesp.br; alekdutra@bol.com.br
}

\section{RESUMO}

A condutividade elétrica na solução de embebição de sementes tem sido um procedimento usado com sucesso para avaliar o vigor de sementes. A presente pesquisa foi desenvolvida com o objetivo de determinar os procedimentos adequados para o teste de condutividade elétrica em sementes de abóbora (Cucurbita moschata Duch ex. Lam). Foram utilizadas sementes do híbrido Bárbara, representadas por cinco lotes ( 1 a 5 ) na primeira etapa e por outros cinco (6 a 10) na segunda. Foram realizados os teste de germinação, primeira contagem de germinação, emergência de plântulas, índice de velocidade de emergência e de condutividade elétrica (CE). Para o teste de CE, foram estudadas variações no volume de água (50 e $75 \mathrm{~mL})$, na temperatura $\left(20,25\right.$ e $\left.30^{\circ} \mathrm{C}\right)$ e no tempo de embebição $(1$, $2,4,8,12,16,20$ e 24 horas). Assim, concluiu-se que a condição mais adequada para o teste de CE é a utilização de 50 sementes em $75 \mathrm{~mL}$ de água por oito horas, à temperatura de $25^{\circ} \mathrm{C}$.

Palavras-chave: Cucurbita moschata, germinação, vigor, potencial fisiológico.

\begin{abstract}
Electrical conductivity of pumpkin hybrid Bárbara seeds

The electrical conductivity in the soaked seed solution has been used successfully to evaluate seed vigor. This work was developed with the objective of determining the adequate procedures to evaluate the electrical conductivity test of pumpkin (Cucurbita moschata Duch ex. Lam) seeds. For pumpkin, hybrid Bárbara, five lots of seeds, in both stages, were used, all with quality patterns (germination) above the minimum demanded by commercialization. The standard germination, first countig of germination, seedling emergency, and emergency speed index electrical conductivity (EC) tests were run. For the EC test two volumes of water $(50$ and $75 \mathrm{~mL})$, three temperatures $\left(20,25\right.$ and $\left.30^{\circ} \mathrm{C}\right)$ and eight period of seed soaking (1, $2,4,8,12,16,20$ and 24 hours) were used. So, it can be concluded that the EC test can be conducted using 50 seeds, $25^{\circ} \mathrm{C}, 75 \mathrm{~mL}$ and $8 \mathrm{~h}$ of embibition period for pumpkin seeds.
\end{abstract}

Keywords: Cucurbita moschata, vigor, germination, physiological potential.

(Recebido para publicação em 31 de março de 2005; aceito em 29 de agosto de 2006)

$\mathrm{O}$ teste padrão de germinação é o procedimento oficial para avaliar a capacidade das sementes produzirem plântulas normais em condições favoráveis de campo, mas nem sempre revela diferenças de qualidade e de desempenho entre lotes de sementes, que podem se manifestar no armazenamento ou mesmo no campo (Carvalho; Nakagawa, 2000). Estudos sobre o potencial fisiológico de diferentes hortaliças como beterraba, tomate, cenoura e ervilha têm evidenciado que o teste de germinação não traduz totalmente o potencial de desempenho de sementes dessas espécies (Nascimento, 1994). Por isso é necessário utilizar testes de vigor, como o teste de condutividade elétrica. Este teste, baseia-se no princípio de que, com o processo de deterioração, ocorre a lixiviação dos constituintes celulares das sementes embebidas em água, devido à perda da integridade dos sistemas celulares. Assim, baixa condutividade significa alta qualidade da semente e alta condutividade, ou seja, maior saída de lixiviados da semente, significa baixa qualidade da semente (Vieira \& Krzyzanowski, 1999;
Panobianco \& Marcos Filho, 2001).

A padronização dos testes de vigor é importante, pois à medida que as técnicas de manejo cultural tornam-se mais sofisticadas, aumenta a necessidade de utilizarem-se sementes de alta qualidade (McDonald, 1998). Os testes de vigor são cada vez mais relevantes para muitas espécies de hortaliças, viabilizando a prática da semeadura de precisão, a eliminação do desbaste e a obtenção da maturação uniforme (Hampton \& Coolbear, 1990).

O presente trabalho foi realizado com o objetivo de determinar procedimentos para realização do teste de condutividade elétrica em sementes de abóbora, visando estabelecer metodologia específica para condução do teste nessa espécie.

\section{MATERIAL E MÉTODOS}

A pesquisa foi desenvolvida no Laboratório de Análise de Sementes do Departamento de Produção Vegetal da UNESP, Campus de Jaboticabal-SP, de agosto a dezembro de 2003. Foram uti- lizadas sementes de abóbora (Cucurbita moschata Duch ex. Lam), híbrido Bárbara (fornecidas pela empresa Sakata Seed Sudamerica Ltda). As sementes foram divididas em cinco lotes (1 a 5) na primeira etapa e outros cinco (6 a 10), na segunda, todos com padrões de qualidade (germinação) acima do mínimo exigido para comercialização. Na segunda etapa foram comparados os procedimentos considerados mais eficientes para o teste, determinados na primeira etapa. Em todo o período experimental, as sementes foram mantidas em embalagens de papel multifoliado e armazenadas em câmara fria $\left(10^{\circ} \mathrm{C}\right.$ e $60 \%$ UR). Foram realizadas as seguintes análises:

Determinação de teor de água (base úmida) realizado em estufa a $105 \pm 3^{\circ} \mathrm{C} / 24 \mathrm{~h}$ (BRASIL, 1992), utilizando-se duas amostras de 20 sementes por lote.

Teste de germinação realizado com quatro repetições de 50 sementes, semeadas em rolos de papel toalha, tipo Germitest, umedecidos com água o equivalente a 2,5 vezes o peso do substrato seco e colocado para germi- 
Tabela 1. Qualidade inicial de dez lotes de sementes de abóbora, híbrido Bárbara ${ }^{1}$. Jaboticabal, UNESP, 2003.

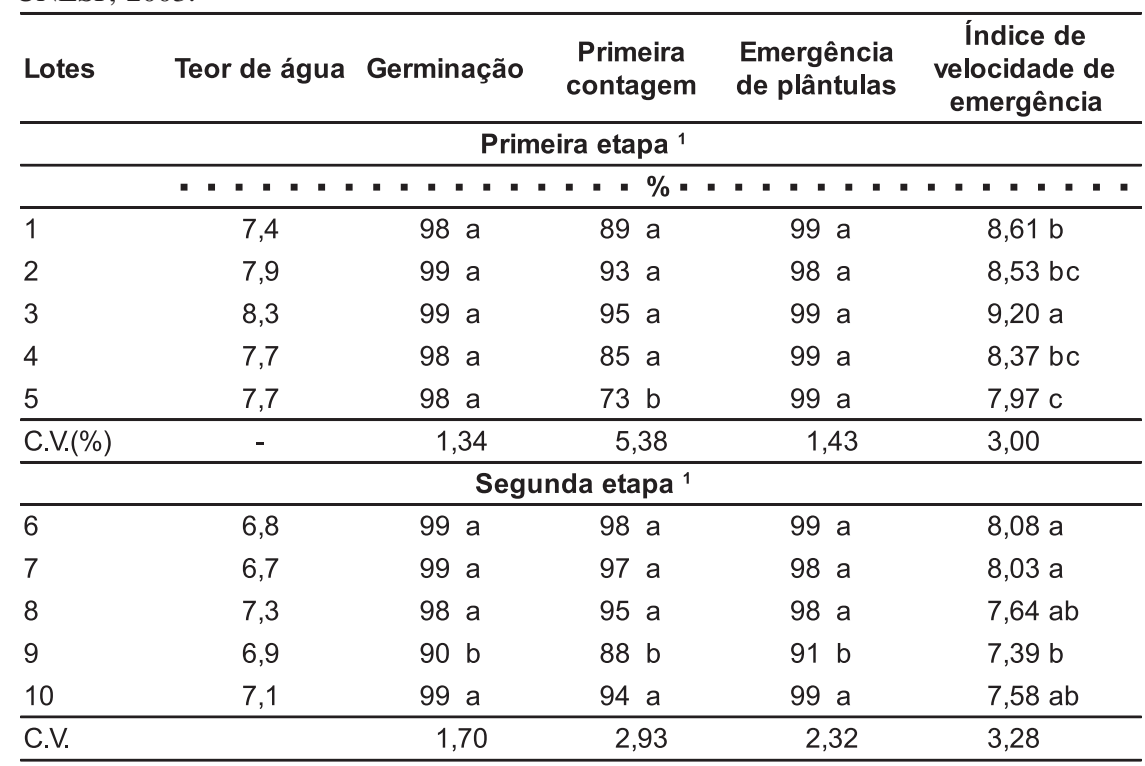

${ }^{1}$ Médias seguidas pela mesma letra, na coluna, em cada etapa, não diferem entre si pelo teste de Tukey a $5 \%$ de probabilidade.

nar a $27^{\circ} \mathrm{C}$. As avaliações foram realizadas quatro e oito dias após a semeadura (ISTA, 1996).

Primeira contagem de germinação conduzida juntamente com o teste padrão de germinação, calculando-se a porcentagem de plântulas normais quatro dias após a semeadura.

Emergência de plântulas avaliada com quatro repetições de 50 sementes, distribuídas em caixas plásticas $(28,5 \times 18,5 \times 10,0 \mathrm{~cm})$, contendo areia de rio como substrato. As caixas foram mantidas em ambiente de laboratório sem controle da temperatura e da umidade relativa do ar. A avaliação foi feita oito dias após a semeadura, considerando o número de plântulas normais.

Índice de velocidade de emergência foram feitas contagens diárias de plântulas emergidas, a partir da instalação do teste (Nakagawa, 1999). Consideraram-se como emergidas as plântulas cujos cotilédones afloraram à superfície da areia. O índice de velocidade de emergência foi calculado utilizando-se fórmula proposta por Maguire (1962).

Teste de condutividade elétrica (CE) foram estudados tempo, temperatura e volume de água de embebição para a condução do teste. Avaliaram-se dois volumes (50 e $75 \mathrm{~mL}$ ) de água, três temperaturas $\left(20,25\right.$ e $\left.30^{\circ} \mathrm{C}\right)$ e oito pe- ríodos $(1,2,4,8,12,16,20$ e 24 horas $)$ de embebição. O teste foi conduzido pelo método de massa, com quatro subamostras de 50 sementes fisicamente puras, pesadas com precisão de duas casas decimais $(0,01 \mathrm{~g})$ e colocadas para embeber (água deionizada) em copos de plásticos (200 mL), mantidos em câmara de germinação, tipo BOD, durante os períodos de embebição. Após o condicionamento, a CE da solução foi medida por meio de leituras em condutivimetro DIGIMED, modelo 21, e os resultados expressos em $\mu \mathrm{S} \mathrm{cm}^{-1} \mathrm{~g}^{-1}$. A leitura foi realizada logo após a retirada do material da incubadora, de modo gradativo, agitando-se cuidadosamente cada recipiente, com o intuito de uniformizar os eletrólitos lixiviados na solução (Hampton \& TeKrony, 1995; Vieira \& Krzyzanowski, 1999).

Os dados obtidos na primeira etapa foram analisados de acordo com o delineamento inteiramente casualizado, no esquema fatorial $5 \times 8$ (cinco lotes e oito períodos de embebição). Cada temperatura e cada volume de água para embebição das sementes foi considerado, para efeito de análise, como um experimento independente. $\mathrm{Na}$ segunda, usou-se fatorial $5 \times 2$ (cinco lotes e dois períodos de embebição). A comparação das médias foi realizada por intermédio do teste de Tukey, 5\% de probabilidade (Banzatto \& Kronka, 1992).

\section{RESULTADOS E DISCUSSÃO}

Os dados do teor de água (TA) das sementes (Tabela 1) foram semelhantes para os cinco lotes componentes de cada etapa com variação de até 0,9 ponto percentual, inferior à amplitude máxima aceita (Marcos Filho, 1999). O vigor, quando avaliado pela primeira contagem (Tabela 1), revelou o lote 5 como de pior qualidade, com os demais lotes componentes da primeira etapa não diferindo entre si. Quando avaliados pelo índice de velocidade de emergência, as sementes do lote 3 mostraram-se mais vigorosas que as demais (Tabela 1). Na segunda etapa, a primeira contagem indicou o lote 9 como o de menor vigor das sementes, resultado corroborado pelo índice de velocidade de emergência (Tabela 1). Nos testes de germinação e de emergência de plântulas não houve diferença entre os lotes estudados, exceto pelo lote 9, da segunda etapa, que foi inferior aos demais (Tabela 1).

Na primeira etapa do estudo, os resultados obtidos com o teste de condutividade elétrica - CE (Tabelas 2 e 3) permitiram verificar que os procedimentos utilizados indicaram pela condutividade elétrica o lote 3 como o mais vigoroso e o lote 2 , como o pior. Para sementes de cebola, o teste de CE não mostrou sensibilidade na separação dos lotes de alto e baixo vigor (Lima, 1993), enquanto para a avaliação do potencial fisiológico das sementes de feijão-de-vagem e de quiabo, o teste de CE foi eficiente, (Dias et al., 1998).

A redução do volume de água, quando se mantiveram constantes os outros fatores (temperatura e período de embebição), estabeleceu relação direta com o aumento do valor da lixiviação (Tabelas 2 e 3). A quantidade de lixiviados, no menor volume, foi em geral superior aos tratamentos com maior volume. Resultados semelhantes foram obtidos com sementes de melão, tendo sido observados valores maiores de lixiviados em menores volumes de água, devido à diluição ocasionada pelo aumento da quantidade de água usada para embebição das sementes (Torres, 2002).

Verificou-se que a elevação da temperatura de $20^{\circ} \mathrm{C}$ para $25^{\circ} \mathrm{C}$ ou $30^{\circ} \mathrm{C}$, 
proporcionou aumento na lixiviação dos exudatos, porém manteve a classificação dos lotes e o acréscimo dos lixiviados durante os períodos de embebição (Tabelas 2 e 3). As várias combinações, quantidade de água/temperatura/período de embebição, indicaram, no geral, aumento progressivo das leituras no intervalo de uma a 24 horas (Tabelas 2 e 3). Resultados similares foram observados em sementes de melão (Torres, 2002).

Após uma hora de embebição todos os tratamentos apresentaram significativa lixiviação, permitindo a distinção dos lotes quanto ao potencial fisiológico. No entanto, a classificação dos lotes foi mais consistente após o período de 8 horas de embebição, o que representa uma significativa redução no período de condicionamento das sementes em relação ao período de 24 horas, adotado pela pesquisa como padrão para testes de CE para ervilha e soja (Hampton \& TeKrony, 1995; Vieira \& Krzyzanowski, 1999). Resultados semelhantes foram observados com sementes de feijão-devagem e de quiabo (Dias et al., 1998) e de tomate (Rodo et al., 1998).

Do ponto de vista da praticidade e de rotina de um laboratório, além de indicações da pesquisa para outras espécies, adotou-se como melhor combinação para a classificação dos lotes de sementes de abóbora, $75 \mathrm{~mL} / 25^{\circ} \mathrm{C}$ (Tabela 3). Em virtude desta constatação, foi conduzida uma segunda etapa, com vistas à confirmação desses resultados, usando cinco outros lotes. Na segunda etapa, o lote 9 foi menos vigoroso que os demais, apresentando valores para germinação de primeira contagem e emergência de plântulas estatisticamente inferiores aos demais (Tabela 1). No índice de velocidade de emergência o lote 9 não diferiu dos lotes 8 e 10, que por sua vez, foram estatisticamente iguais aos lotes 6 e 7 (Tabela 1).

Quando avaliou-se a CE, o tempo de embebição de 8 horas definiu maior número de classes de vigor que 24 horas (Tabela 4). Ao contrário dos testes de avaliação de qualidade inicial dos lotes (Tabela 1), a análise de CE foi eficiente em identificar o lote mais vigoroso, neste caso, o lote 7 (Tabela 4). O teste de CE já havia sido identificado
Tabela 2. Condutividade elétrica $\left(\mu \mathrm{S} \mathrm{cm}^{-1} \mathrm{~g}^{-1}\right.$ ) de sementes de abóbora, híbrido Bárbara, em função dos períodos de embebição e dos lotes, utilizando-se $50 \mathrm{~mL}$ de água ${ }^{1}$. Jaboticabal, UNESP, 2003.

\begin{tabular}{|c|c|c|c|c|c|c|c|c|}
\hline \multirow{2}{*}{ Lotes } & \multicolumn{8}{|c|}{ Períodos de embebição (h) } \\
\hline & 1 & 2 & 4 & 8 & 12 & 16 & 20 & 24 \\
\hline \multicolumn{9}{|c|}{$20^{\circ} \mathrm{C}^{1}$} \\
\hline 1 & $53,1 \mathrm{c}$ & $61,1 \mathrm{~b}$ & $64,7 \mathrm{~b}$ & $71,4 \mathrm{~b}$ & $72,2 \mathrm{~b}$ & $79,8 \mathrm{c}$ & $79,9 \mathrm{~b}$ & $87,4 \mathrm{a}$ \\
\hline 2 & $75,2 \mathrm{a}$ & 83,6 a & $88,7 \mathrm{a}$ & 86,8 a & $82,0 \mathrm{a}$ & $101,0 \mathrm{a}$ & $105,4 \mathrm{a}$ & $92,2 \mathrm{a}$ \\
\hline 3 & $21,4 d$ & $33,0 \mathrm{~d}$ & $40,2 \mathrm{c}$ & $40,1 \mathrm{~d}$ & $40,7 \mathrm{c}$ & $37,7 \mathrm{e}$ & $36,1 \mathrm{~d}$ & $31,6 \mathrm{~d}$ \\
\hline 4 & $58,2 \mathrm{c}$ & $57,8 \mathrm{~b}$ & $64,4 \mathrm{~b}$ & $67,6 \mathrm{c}$ & $80,2 \mathrm{a}$ & $69,0 \mathrm{~d}$ & $65,2 \mathrm{c}$ & $64,6 \mathrm{c}$ \\
\hline 5 & $66,2 \mathrm{~b}$ & $49,4 \mathrm{c}$ & $86,2 \mathrm{a}$ & $74,6 \mathrm{~b}$ & $83,2 \mathrm{a}$ & $86,4 \mathrm{~b}$ & $78,4 \mathrm{~b}$ & $74,0 \mathrm{~b}$ \\
\hline \multicolumn{9}{|c|}{ C.V. $(\%)=4,58$} \\
\hline \multicolumn{9}{|c|}{$25^{\circ} \mathrm{C}^{1}$} \\
\hline 1 & $62,5 \mathrm{c}$ & $44,9 d$ & $52,6 \mathrm{~d}$ & $80,7 \mathrm{~b}$ & $85,6 \mathrm{~b}$ & $94,7 \mathrm{~b}$ & $101,7 \mathrm{~b}$ & $99,1 \mathrm{~b}$ \\
\hline 2 & $91,3 \mathrm{a}$ & 104,4 a & 103,4 a & 92,2 a & 95,9 a & $106,9 a$ & 111,2 a & $105,9 a$ \\
\hline 3 & $36,3 d$ & $38,9 \mathrm{~d}$ & $40,0 \mathrm{~d}$ & $41,2 \mathrm{c}$ & $40,4 d$ & $47,5 d$ & $47,2 \mathrm{~d}$ & $40,5 \mathrm{~d}$ \\
\hline 4 & $68,4 \mathrm{c}$ & $64,2 \mathrm{c}$ & $64,8 \mathrm{c}$ & $80,5 \mathrm{~b}$ & $82,2 \mathrm{~b}$ & $81,7 \mathrm{c}$ & $77,7 \mathrm{c}$ & $86,8 \mathrm{c}$ \\
\hline 5 & $75,3 \mathrm{~b}$ & $74,4 \mathrm{~b}$ & $88,9 \mathrm{~b}$ & 89,9 a & $74,9 \mathrm{c}$ & $98,4 \mathrm{~b}$ & $97,9 \mathrm{~b}$ & $87,8 \mathrm{c}$ \\
\hline \multicolumn{9}{|c|}{ C.V. $(\%)=4,01$} \\
\hline \multicolumn{9}{|c|}{$30^{\circ} \mathrm{C}^{1}$} \\
\hline 1 & $71,8 \mathrm{~b}$ & $76,1 \mathrm{~b}$ & $85,6 \mathrm{~b}$ & $97,9 \mathrm{~b}$ & $101,9 a$ & $108,6 \mathrm{~b}$ & $108,5 \mathrm{a}$ & $112,3 a$ \\
\hline 2 & $86,1 \mathrm{a}$ & 97,5 a & $114,0 \mathrm{a}$ & 104,2 a & 103,8 a & $123,3 \mathrm{a}$ & 113,8 a & $112,9 a$ \\
\hline 3 & $42,1 \mathrm{c}$ & $38,1 \mathrm{~d}$ & $43,9 \mathrm{c}$ & $45,6 d$ & $47,3 d$ & $41,6 \mathrm{e}$ & $48,0 \mathrm{c}$ & $41,9 \mathrm{~d}$ \\
\hline 4 & $70,4 \mathrm{~b}$ & $66,6 \mathrm{c}$ & $82,1 \mathrm{~b}$ & $73,5 \mathrm{c}$ & $83,2 \mathrm{c}$ & $80,1 \mathrm{~d}$ & $80,1 \mathrm{~b}$ & $85,1 \mathrm{c}$ \\
\hline 5 & $70,9 \mathrm{~b}$ & $81,6 \mathrm{~b}$ & $83,7 \mathrm{~b}$ & $93,4 \mathrm{~b}$ & $94,3 \mathrm{~b}$ & $97,2 \mathrm{c}$ & 108,2 a & $103,1 \mathrm{~b}$ \\
\hline
\end{tabular}

${ }^{1}$ Médias seguidas pela mesma letra, na coluna para cada temperatura, não diferem entre si pelo teste de Tukey a $5 \%$ de probabilidade.

Tabela 3. Condutividade elétrica $\left(\mu \mathrm{S} \mathrm{cm}^{-1} \mathrm{~g}^{-1}\right)$ de sementes de abóbora, híbrido Bárbara, em função dos períodos de embebição e lotes, utilizando-se 75mL de água ${ }^{1}$. Jaboticabal, UNESP, 2003.

\begin{tabular}{|c|c|c|c|c|c|c|c|c|}
\hline \multirow{2}{*}{ Lotes } & \multicolumn{8}{|c|}{ Períodos de embebição (h) } \\
\hline & 1 & 2 & 4 & 8 & 12 & 16 & 20 & 24 \\
\hline \multicolumn{9}{|c|}{$20^{\circ} \mathrm{C}^{1}$} \\
\hline 1 & $37,7 \mathrm{c}$ & $41,6 \mathrm{~b}$ & $44,8 \mathrm{~b}$ & $51,4 \mathrm{a}$ & $53,4 \mathrm{~b}$ & $54,0 \mathrm{~b}$ & $56,3 \mathrm{~b}$ & $58,2 \mathrm{~b}$ \\
\hline 2 & $53,1 \mathrm{a}$ & 51,6 a & $55,4 a$ & 56,4 a & 59,4 a & 63,3 a & $64,0 \mathrm{a}$ & 67,2 a \\
\hline 3 & $22,7 d$ & $22,2 \mathrm{c}$ & $23,9 \mathrm{c}$ & $25,4 \mathrm{c}$ & $29,4 \mathrm{c}$ & $27,3 d$ & $29,1 d$ & $31,0 \mathrm{~d}$ \\
\hline 4 & $43,6 \mathrm{~b}$ & $50,2 a$ & $46,1 \mathrm{~b}$ & $44,8 \mathrm{~b}$ & $51,8 \mathrm{~b}$ & $48,7 \mathrm{c}$ & $51,4 \mathrm{c}$ & $44,5 \mathrm{c}$ \\
\hline 5 & $45,0 \mathrm{~b}$ & $49,2 \mathrm{a}$ & $53,9 \mathrm{a}$ & $53,3 \mathrm{a}$ & $61,9 a$ & $57,6 \mathrm{~b}$ & $60,5 \mathrm{a}$ & $56,3 \mathrm{~b}$ \\
\hline \multicolumn{9}{|c|}{ C.V. $(\%)=5,82$} \\
\hline \multicolumn{9}{|c|}{$25^{\circ} \mathrm{C}^{1}$} \\
\hline 1 & $41,8 \mathrm{~b}$ & $47,5 \mathrm{~b}$ & $49,3 \mathrm{~b}$ & $56,3 a$ & $58,7 \mathrm{~b}$ & $63,4 \mathrm{~b}$ & $61,3 b$ & $67,4 \mathrm{~b}$ \\
\hline 2 & $57,4 \mathrm{a}$ & $60,4 a$ & $63,2 a$ & $60,0 \mathrm{a}$ & $67,5 \mathrm{a}$ & 69,6 a & $76,5 \mathrm{a}$ & 79,5 a \\
\hline 3 & $23,6 \mathrm{c}$ & $26,5 \mathrm{c}$ & $28,0 \mathrm{c}$ & $26,7 \mathrm{c}$ & $28,4 d$ & $31,7 d$ & $31,9 d$ & $27,6 \mathrm{~d}$ \\
\hline 4 & $41,8 \mathrm{~b}$ & $42,8 \mathrm{~b}$ & $46,9 \mathrm{~b}$ & $47,0 \mathrm{~b}$ & $49,7 \mathrm{c}$ & $52,1 \mathrm{c}$ & $55,5 \mathrm{c}$ & $46,9 \mathrm{c}$ \\
\hline 5 & $56,7 \mathrm{a}$ & $58,0 \mathrm{a}$ & $61,9 \mathrm{a}$ & $58,0 \mathrm{a}$ & $58,4 \mathrm{~b}$ & $66,5 \mathrm{~b}$ & $61,7 \mathrm{~b}$ & $72,8 \mathrm{~b}$ \\
\hline \multicolumn{9}{|c|}{ C.V. $(\%)=5,82$} \\
\hline \multicolumn{9}{|c|}{$30^{\circ} \mathrm{C}^{1}$} \\
\hline$\overline{1}$ & $49,9 \mathrm{~b}$ & $54,9 a$ & $59,7 \mathrm{a}$ & $66,4 \mathrm{~b}$ & $67,1 \mathrm{~b}$ & $76,3 \mathrm{a}$ & $78,4 \mathrm{~b}$ & $79,7 \mathrm{a}$ \\
\hline 2 & $64,1 \mathrm{a}$ & $56,5 \mathrm{a}$ & $63,3 a$ & $80,8 \mathrm{a}$ & $73,0 \mathrm{~b}$ & $75,0 \mathrm{a}$ & 91,9 a & 85,9 a \\
\hline 3 & $25,9 \mathrm{c}$ & $28,2 \mathrm{~b}$ & $30,6 \mathrm{c}$ & $33,0 \mathrm{~d}$ & $33,4 \mathrm{~d}$ & $35,1 \mathrm{c}$ & $34,1 \mathrm{~d}$ & $27,8 \mathrm{~d}$ \\
\hline 4 & $48,3 \mathrm{~b}$ & $51,4 a$ & $43,2 \mathrm{~b}$ & $56,0 \mathrm{c}$ & $55,6 \mathrm{c}$ & 56,7 b & $62,8 \mathrm{c}$ & $57,7 \mathrm{c}$ \\
\hline 5 & $59,8 \mathrm{a}$ & $53,7 \mathrm{a}$ & $61,5 \mathrm{a}$ & $62,4 \mathrm{~b}$ & $80,4 \mathrm{a}$ & $73,3 \mathrm{a}$ & $67,9 \mathrm{c}$ & $65,9 \mathrm{~b}$ \\
\hline \multicolumn{9}{|c|}{ C.V. $(\%)=5,71$} \\
\hline
\end{tabular}

${ }^{1}$ Médias seguidas pela mesma letra, na coluna para cada temperatura não diferem entre si pelo teste de Tukey a $5 \%$ de probabilidade de erro. 
Tabela 4. Condutividade elétrica de sementes de abóbora, híbrido Bárbara, em função dos períodos de embebição e dos lotes, usando-se $75 \mathrm{~mL} / 25^{\circ} \mathrm{C}^{1}$. Jaboticabal, UNESP, 2003.

\begin{tabular}{|c|c|c|}
\hline \multirow{3}{*}{ Lotes } & \multicolumn{2}{|c|}{ Períodos de embebição (h) } \\
\hline & 8 & 24 \\
\hline & $\because . . . . . .$. & $\mu \mathbf{S ~ c m}^{-1} \mathbf{g}^{-1} \cdot \ldots$ \\
\hline 6 & $62,7 \mathrm{c}$ & $76,0 \mathrm{~b}$ \\
\hline 7 & $50,7 d$ & $59,1 \mathrm{c}$ \\
\hline 8 & 79,0 a & $88,2 \mathrm{a}$ \\
\hline 9 & $67,6 \mathrm{bc}$ & $82,2 \mathrm{ab}$ \\
\hline 10 & $71,7 \mathrm{~b}$ & $84,2 \mathrm{a}$ \\
\hline
\end{tabular}

${ }^{1}$ Médias seguidas pela mesma letra, na coluna, não diferem entre si pelo teste de Tukey a 5\% de probabilidade.

como promissor para avaliação do potencial fisiológico em sementes de quiabo (Dias et al., 1998) e tomate (Sá, 1999), embora em alface o teste não tenha sido eficiente (Franzin et al., 2004).

Várias combinações de volume, período e temperatura de embebição das sementes foram utilizadas. Dentre elas, a embebição das sementes em $75 \mathrm{~mL}$ de água, por 8 horas a $25^{\circ} \mathrm{C}$ nas condições deste trabalho foi a mais afetada. Os resultados obtidos neste trabalho indicam que o teste de CE tem potencial para ser utilizado como indicador do vigor de lotes de sementes de abóbora.

\section{REFERÊNCIAS}

BANZATTO DA; KRONKA SN. 1992. Experimentação agrícola. 2. ed. Jaboticabal: FUNEP/ UNESP. 247p.
BRASIL, Ministério da Agricultura. 1992. Regras para análise de sementes. Brasília: SNAD/ CLAV. 365p.

CARVALHO NM; NAKAGAWA J. 2000. Sementes: ciência, tecnologia e produção. 4 ed Jaboticabal: FUNEP/UNESP. 424p.

DIAS DCFS; VIEIRAAN; BHÉRING MC. 1998. Condutividade elétrica e lixiviação de potássio para avaliação do vigor de sementes de hortaliças: feijão-de-vagem e quiabo. Revista Brasileira de Sementes 20: 408-413.

FRANZIN SM; MENEZES NL; GARCIA DC; WRASSE CF. 2004. Métodos para avaliação do potencial fisiológico de sementes de alface. Revista Brasileira de Sementes 26: 63-69.

HAMPTON JG; COOLBEAR P. 1990. Potential versus actual seed performance can vigour testing provide an answer? Seed Science and Technology 18: 215-228.

HAMPTON JG; TEKRONY DM. 1995. Handbook of vigor test methods. Zurich: ISTA. $117 \mathrm{p}$.

INTERNATIONAL SEED TESTING ASSOCIATION (ISTA). 1996. International Rules for Seed Testing. Seed Science and Technology 24: 335. Supplement.
LIMA D. 1993. Avaliação da viabilidade e vigor de sementes de cebola (Allium cepa L.). Pelotas: UFPel. 61p. (Dissertação).

MAGUIRE JD. 1962. Speed of germination-aid selection and evaluation for seedling emergence and vigor. Crop Science, Madison 2: 176-177.

MARCOS FILHO J. 1999. Teste de envelhecimento acelerado. In: KRZYZANOWSKI FC; VIEIRA RD; FRANÇA NETO JB (eds). Vigor de sementes: conceitos e testes. Londrina: ABRATES. p.1-24.

MCDONALD MB. 1998. Improving our understanding of vegetable and flower seed quality. Seed Technology 20: 121-124.

NAKAGAWA J. 1999. Testes de vigor baseados no desempenho das plântulas. In: KRZYZANOWSKI FC; VIEIRA RD; FRANÇA NETO JB (eds). Vigor de sementes: conceitos e testes. Londrina: ABRATES. cap 2. p.1-24.

NASCIMENTO WM. 1994. A importância da qualidade de semente olerícolas. A lavoura. Rio de Janeiro. p.38-39.

PANOBIANCO M; MARCOS FILHO J. 2001. Envelhecimento acelerado e deterioração controlada em sementes de tomate. Scientia Agrícola 58 : 525-531.

RODO AB; TILLMANN MAA; VILLELA FA; SAMPAIO NV. 1998. Teste de condutividade elétrica em sementes de tomate. Revista Brasileira de Sementes 20: 29-38.

SÁ ME. 1999. Condutividade elétrica em sementes de tomate (Lycopersicum lycopersicum L.). Scientia Agrícola 56: 13-19.

TORRES SB. 2002. Métodos para avaliação do potencial fisiológico de sementes de melão. Piracicaba: USP-ESALQ. 103p. (Tese).

VIEIRA RD, KRZYZANOWSKI FC. 1999. Teste de condutividade elétrica. In: KRZYZANOWSKI FC; VIEIRA RD; FRANÇA NETO JB (eds). Vigor de sementes: conceitos e testes. Londrina: ABRATES. cap.4. p.1-26. 